\title{
ALTERAÇÃO DE BIOMARCADORES DE LESÃO MIOCÁRDICA EM ATLETAS APÓS A MARATONA INTERNACIONAL DE SÃO PAULO
}

\author{
CHANGES IN BIOMARKERS OF MYOCARDIAL LESION IN RUNNERS AFTER THE SÃO PAULO \\ INTERNATIONAL MARATHON
}

\section{ALTERACIÓN DE BIOMARCADORES DE LESIÓN MIOCÁRDICA EN ATLETAS DESPUÉS DE MARATÓN INTERNACIONAL DE SAN PABLO}

\author{
Ana Paula Sierra1,2,3 \\ (Educadora Fisica) \\ Nabil Ghorayeb 3 (Médico) \\ Giuseppe Sebastiano Dioguardi ${ }^{3}$ \\ (Médico) \\ Carlos Anibal Sierra ${ }^{4}$ (Médico) \\ Maria Augusta Peduti Dal Molin Kiss \\ (Médica) \\ 1. Universidade de São Paulo, São \\ Paulo, Escola de Educação Física e \\ Esporte, São Paulo, Brasil. \\ 2. Universidade Nove de Julho, \\ Departamento de Medicina, São \\ Paulo, São Paulo, Brasil. \\ 3. Instituto Dante Pazzanese de \\ Cardiologia, Seção de Cardioesporte, \\ São Paulo, São Paulo, Brasil. \\ 4. Instituto Dante Pazzanese de \\ Cardiologia, Setor de Eletrofisiologia, \\ São Paulo, São Paulo, Brasil.
}

\section{Correspondência}

Av. Professor Mello de Moraes, 65, Cidade Universitária, São Paulo, SP, Brasil. 05508-030.

anasierra@usp.br

\section{RESUMO}

Introdução: Apesar de a prática regular de exercício físico reduzir o risco de doença cardiovascular, estudos recentes têm documentado elevações em biomarcadores relacionados com danos cardíacos após exercícios prolongados em indivíduos aparentemente saudáveis. Objetivo: Investigar as alterações nos níveis de brain natriuretic peptide (BNP) e troponina T cardíaca (CTnT) em atletas amadores após uma maratona, assim como verificar as possíveis relações entre as alterações, antes e depois da prova, apresentada pelos dois biomarcadores e variáveis do teste cardiopulmonar. Métodos: Estudamos 38 atletas do sexo masculino $(40,9 \pm 6,29$ anos) antes e depois da Maratona Internacional de São Paulo, SP, Brasil. Foram realizadas coletas de sangue na veia antecubital para mensurar os biomarcadores cardíacos, cTnT e BNP 24h antes, imediatamente após e $24 \mathrm{~h}$ após a maratona. Foi realizado teste cardiopulmonar máximo nas três semanas que antecederam a prova. Resultados: Os valores de BNP e cTnT aumentaram imediatamente após a maratona $(p<0,001)$ quando comparados com os valores basais. No terceiro momento (24h) os valores de troponina tiveram uma redução significativa caracterizando um retorno aos valores basais. Não encontramos correlação entre idade e variáveis referentes a intensidade da maratona, porém encontramos correlação dos biomarcadores com o tempo de conclusão da maratona. Conclusão: Diferentes causas de liberação podem ser assumidas para cTnT e BNP e, neste caso, parecem não refletir dano miocárdico devido ao comportamento da curva destes marcadores, além de não haver relação entre a liberação dos dois biomarcadores.

Palavras-chave: troponina t, marcadores biológicos, peptídeo natriurético encefálico, resistência física.

\section{ABSTRACT}

Introduction: Although the practice of physical exercises reduces the cardiovascular risk, recent studies have documented elevations in cardiac injury biomarkers after prolonged exercises in apparently healthy individuals. Objective: We aimed to investigate the changes in brain natriuretic peptide (BNP) and cardiac troponin T (CTnT) levels after a marathon, and the correlation of these biomarkers and the variables of cardiopulmonary test. Methods: We studied 38 male athletes ( $40.9 \pm 6.29$ years old) participants at the São Paulo International Marathon, SP, Brasil. Blood collections were performed to measure cTnT and BNP 24h before, immediately after and $24 \mathrm{~h}$ after the marathon. Cardiopulmonary test was performed in the three weeks preceding the marathon. Results: BNP and cTnT values increased significantly after the marathon $(p<0.001)$. In the third moment (24h) the troponin values returned to the baseline values. We found no correlation between age and variables of the marathon, however, we did find a correlation between the biomarkers and time to complete the marathon. Conclusion: Various causes of release may be presumed for CTnT and BNP, however, they do not seem to reflect into myocardial injury, and there is no relationship between the releases of the two biomarkers.

Keywords: troponin t, biological markers, natriuretic peptide, brain physical endurance.

\section{RESUMEN}

Introducción: A pesar de que la práctica regular de ejercicio reduce el riesgo de enfermedad cardiovascular, estudios recientes han documentado elevaciones en biomarcadores relacionados con daños cardiacos después de ejercicios prolongados en individuos aparentemente saludables. Objetivo: Investigar las alteraciones en los niveles de BNP y troponina T cardiaca (CTnT) en atletas amateur después de maratón, así como verificar las posibles relaciones entre las alteraciones, antes y después de la prueba, presentada por dos biomarcadores y variables del test

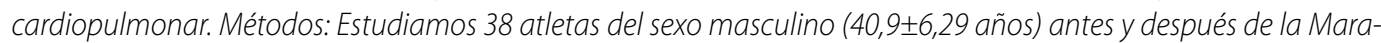
tón Internacional de São Paulo, SP, Brasil. Fueron realizadas colectas de sangre en la vena antecubital para medir los biomarcadores cardiacos, CTnT y BNP, 24 horas antes, inmediatamente y 24 horas después de la Maratón. Fue realizado test cardiopulmonar máximo en las tres semanas que antecedieron a la maratón. Resultados: Los valores de BNP y cTnT aumentaron inmediatamente después de la maratón $(p<0,001)$ cuando comparados con los valores 
basales. En el tercer momento (24h) los valores de troponina tuvieron una reducción significativa caracterizando un retorno a los valores basales. No encontramos correlación entre edad y variables referentes a intensidad de la maratón, sin embargo encontramos correlación de los biomarcadores con el tiempo de conclusión de la maratón. Conclusión: Diferentes causas de liberación pueden ser asumidas para CTnTy BNP y, en este caso, parecen no reflejar daño miocárdico debido al comportamiento de la curva de estos marcadores, además de no haber relación entre la liberación de los dos biomarcadores.

Palabras clave: troponina t, marcadores biológicos, péptido natriurético encefálico, resistencia física.

\section{INTRODUÇÃO}

Nas últimas décadas têm sido relatado um crescimento elevado no número de provas e de participantes no segmento de corrida de rua principalmente nas corridas de longa distância ${ }^{1-3}$.

A maratona é uma modalidade de corrida realizada por participantes com diferentes níveis de condicionamento, ampla distribuição por idade e com a presença de diversas comorbidades ${ }^{4}$.

A prática esportiva requer adaptações neuro-imuno-endócrinas para manter a homeostase, durante o repouso e principalmente durante o exercício. Essas adaptações influenciam a atividade do sistema cardiovascular, respiratório e musculoesquelético ${ }^{5}$. Já é bem estabelecido na literatura que o exercício físico aeróbico traz benefícios ao sistema cardiovascular, entretanto ainda não há um consenso quanto aos efeitos do exercício de longa distância na promoção da saúde ou no risco cardiovascular.

Estudos recentes demonstraram elevações em níveis séricos de biomarcadores relacionados a danos cardíacos, após exercícios prolongados, de intensidade moderada, como maratona, ultramaratona e triathlon, em indivíduos aparentemente saudáveis ${ }^{6,7}$.

As troponinas são marcadores altamente específicos e sensíveis ao dano dos cardiomiócitos, consideradas de grande eficácia no diagnóstico de síndromes coronarianas agudas ${ }^{8}$. Concentrações elevadas de troponina após exercícios de longa duração têm sido encontradas, porém, sem a presença concomitante de sintomas clínicos de infarto agudo do miocárdio ${ }^{7-10}$. Os mecanismos e a relevância deste aumento para a prática clínica, assim como os fatores associados ainda não estão claros.

Outro marcador sintetizado pelos cardiomiócitos ventriculares é o brain natriuretic peptide (BNP). Os níveis de BNP estão aumentados em resposta ao aumento da tensão parietal por disfunção sistólica e/ou diastólica ou pelo estiramento das fibras ventriculares causado pela sobrecarga de volume e/ou pressão em doenças como hipertrofia ventricular esquerda, cardiomiopatias e insuficiência cardíaca, com elevação proporcional à gravidade da alteração estrutural e hemodinâmica. Entretanto, as concentrações de BNP não se elevam em corações fisiologicamente hipertrofiados, como o "coração de atleta"1,11,12.

Estudos recentes demonstram que existe um aumento nos níveis de BNP, após exercícios prolongados em indivíduos aparentemente saudáveis. Da mesma forma, o significado do aumento de BNP, bem como a relação com as troponinas, após exercício prolongado, permanece sem resposta1,13.

Neste contexto, esse estudo objetivou investigar as modificações nos níveis de BNP e troponina cardíaca em maratonistas após maratona, além de tentar estabelecer uma relação entre as alterações desses biomarcadores com características gerais dos atletas e da prova.

\section{MÉTODOS}

Entre Abril e Maio de 2006 foram recrutados, utilizando um email convite para os maratonistas das principais assessorias esportivas de São Paulo, que tinham como objetivo a participação na Maratona Internacional de São Paulo, SP, Brasil, que ocorreu no final do mês de Maio de 2006.
Dentre os respondentes, 68 atletas maratonistas se apresentaram tendo sido avaliados no ambulatório da Seção de CardioEsporte do Instituto Dante Pazzanese de Cardiologia, SP, Brasil e convidados a participar do estudo. Entretanto, 30 sujeitos não completaram todas as etapas da pesquisa, sendo excluídos da amostra final.

Foram estudados 38 atletas amadores do sexo masculino (idade

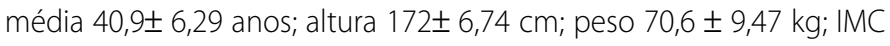
23,46 $\pm 2,24 \mathrm{~kg} / \mathrm{m}^{2}$ e VO2 48,75 \pm 4,62 ml/kg/min) em três momentos: antes, imediatamente após e 24 horas após a participação na Maratona Internacional de São Paulo (42,195km), realizada em 2006.

Após contato inicial, quando foram apresentados os objetivos, possíveis benefícios, riscos e o protocolo do estudo, todos os participantes assinaram o Termo de Consentimento Livre e Esclarecido do estudo previamente aprovado pelo Comitê de Ética em Pesquisa do Instituto Dante Pazzanese de Cardiologia.

Com o objetivo de diagnosticar doenças agudas ou crônicas, durante o período de seleção dos participantes, foi realizada anamnese, exame físico, um eletrocardiograma de repouso e teste cardiopulmonar.

Não houve intervenções na rotina de treino ou alimentar durante o período do estudo. Para inclusão no estudo havia a obrigatoriedade do atleta ter participado de, pelo menos, uma maratona nos últimos cinco anos e/ou uma meia-maratona no último ano.

Com o objetivo de determinar as concentrações dos biomarcadores cardíacos, amostras de sangue periférico foram coletadas através da veia antecubital, 24 horas antes, imediatamente e 24 horas após a participação de todos os sujeitos na Maratona Internacional de São Paulo, SP, Brasil.

\section{Biomarcadores cardíacos}

Amostras de plasma, obtidas após centrifugação do sangue periférico, foram utilizadas para quantificação do brain natriuretic peptide (BNP). A sensibilidade do teste é 1 (um) pg/mL e o limite superior de referência para sujeitos saudáveis até $100 \mathrm{pg} / \mathrm{mL}$.

A troponina $\mathrm{T}$ (cTnT) foi avaliada nas amostras de plasma por quimioluminescência amplificada com analisador automatizado, detectando troponina cardíaca livre bem como complexos binários e terciários. Para cTnT, a cross reatividade com a troponina T esquelética e humana cTnT é de 0,001 e 0,002\%, respectivamente. Os limites superiores de referência definidos em sujeitos saudáveis é $<0,01 \mu \mathrm{g} / \mathrm{L}$ para cTnT.

Foi realizado um teste cardiopulmonar máximo em esteira rolante acoplada a analisador de gases da marca Medgraphics, durante as três semanas que antecederam a maratona. O protocolo utilizado foi em rampa, iniciando com oito $\mathrm{km} / \mathrm{h}$, e aumento a cada um minuto de um km/h.

\section{Análise estatística}

Para os cálculos estatísticos foi utilizado o software SPSS versão 19.0 (SPSS Inc., USA, 2010). Os dados são apresentados em estatísticas descritivas de média, desvio padrão e quartis (mediana, percentil 25 e percentil 75). 
Foi realizado o teste de Kolmogorov-Smirnov para testar a normalidade das variáveis, as quais rejeitaram a normalidade. Desta forma, os resultados estão expressos também em mediana e intervalos interquartis, para melhor visualização da tendência central das amostras.

Para avaliar o impacto da maratona nos dados obtidos nos três momentos estudados para a troponina foi realizada ANOVA não paramétrica; para os dois momentos do BNP, o teste não paramétrico de Wilcoxon e, para verificar as correlações entre as variáveis, o Rho de Spearman ${ }^{14}$.

As medidas dos marcadores também foram categorizadas quanto ao seu percentual alterado nos três momentos e uma análise não paramétrica de dados ordinais com medidas repetidas foi realizado ${ }^{15}$. O nível de significância foi estabelecido em 5\%.

\section{RESULTADOS}

A Maratona Internacional de São Paulo foi realizada durante o outono. A prova se iniciou com $15,8^{\circ} \mathrm{C}$ e $85 \%$ de umidade relativa do ar e $0 \mathrm{~m} / \mathrm{s}$ de velocidade do vento. No final da maratona a temperatura era de $25,7^{\circ} \mathrm{C}, 44 \%$ de umidade relativa do ar e $2 \mathrm{~m} / \mathrm{s}$ de velocidade do vento. $\mathrm{O}$ dia manteve-se ensolarado durante todo o período da maratona. O tempo médio de prova realizado pelos atletas avaliados foi de 03:58:37 $\pm 00: 43: 36$ horas, com velocidade media de 10,64km/h, repre-

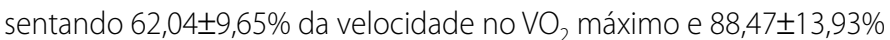
da velocidade no limiar anaeróbio.

Durante e depois da corrida, nenhum atleta apresentou sinais ou sintomas que sugerissem doença aguda, de acordo com avaliação clínica.

\section{Níveis de BNP e cTnT}

Os valores plasmáticos de BNP e cTnT, nos três momentos, assim como a diferença entre os valores pré e pós maratona são mostrados na tabela 1.

Conforme apresentado na tabela 1, os valores de BNP e cTnT obtidos imediatamente após o exercício aumentaram significativamente ( $p<0,001$ e $p=0,0033$, respectivamente) comparados aos valores iniciais. Os valores de troponina, observados 24 horas após a maratona, mostraram redução significativa em relação aos valores imediatamente depois da prova, caracterizando um retorno aos valores basais.

Não foram realizadas análises de BNP 24 horas após a maratona. A curva individual de BNP e cTnT dos participantes está apresentada na figura 1.

Tabela 1. Valores dos biomarcadores: BNP e Troponina antes, depois e 24 horas após a Maratona Internacional de São Paulo.

\begin{tabular}{c|c|c|c|c|c}
\hline Variável & Pré & Pós & 24h & $\begin{array}{c}\text { Diferença } \\
\text { (pós - pré) }\end{array}$ & $\begin{array}{c}\text { Valor } \\
\text { de } \mathbf{p}\end{array}$ \\
\hline $\begin{array}{c}\text { Troponina } \\
(\mathrm{ng} / \mathrm{mL})\end{array}$ & $\begin{array}{c}0,0016 \pm 0,007 \\
0(0 ; 0)\end{array}$ & $\begin{array}{c}0,0726 \pm 0,279 \\
0(0 ; 0,01)\end{array}$ & $\begin{array}{c}0,0195 \pm 0,079 \\
0(0 ; 0)\end{array}$ & $\begin{array}{c}0,0711 \pm 0,278 \\
0(0 ; 0,01)\end{array}$ & 0,0033 \\
\hline $\mathrm{BNP}$ & $10,1 \pm 23,46$ & $67,2 \pm 74,6$ & & $57,1 \pm 69,5$ & \\
$(\mathrm{pg} / \mathrm{mL})$ & $0(0 ; 8,9)$ & $44,4(23,9 ; 78,1)$ & & $39,7(16 ; 76,7)$ & $<0,001$ \\
\hline
\end{tabular}

Abreviações: Pré, pré maratona; pós, pós maratona; 24 h, 24 horas após a maratona; Os valores săo expressos em: media \pm desvio padrão e mediana (percentil 25; percentil 75).

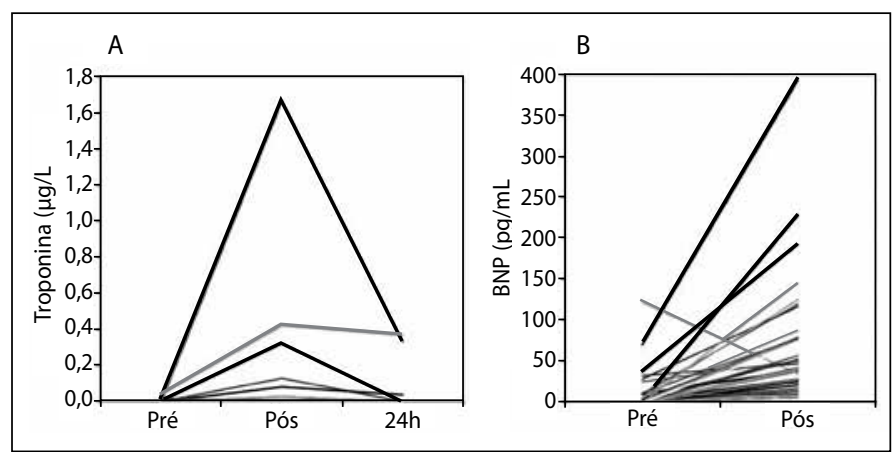

Figura 1. Distribuição do hábito de frequentar academia entre os atletas, segundo presença de lesão na temporada de 2014.
Antes da maratona, os valores de BNP de todos os atletas, exceto um, estavam dentro da variação normal, abaixo de 100pg/mL. Porém, imediatamente após a maratona, sete atletas $(18,4 \%)$ tinham valores de BNP acima dos limites de referência de $100 \mathrm{pg} / \mathrm{mL}$.

Em relação à troponina, dois atletas demonstraram valores alterados antes da maratona, 13 atletas imediatamente após e três atletas 24 horas após a maratona, mostrando diferença significativa entre os três momentos estudados (tabela 2).

Não foram observadas correlações significativas entre as diferenças dos valores: pós e pré maratona para a troponina e BNP $(r=0,226$, $P=0,173)$, assim como, entre os valores imediatamente após maratona de ambos marcadores $(r=0,196, P=0,237)$. Também não houve correlação entre as diferenças do BNP $(r=0,225, p=0,175)$ e troponina $(r=0,173$, $\mathrm{p}=0,299)$ com a idade e \% da velocidade no limiar anaeróbio e pico. Entretanto, verificou-se correlação positiva e moderada do tempo de conclusão da maratona com as diferenças entre os valores pós e pré, e os valores imediatamente após a maratona, tanto do BNP como da troponina ( $r=0,408, p=0,011$ e $r=0,375, p=0,021)$, demonstrando que quanto mais tempo de exercício maior a diferença entre os valores pré e pós dos dois biomarcadores.

Tabela 2. Frequência da alteração: BNP e Troponina antes, depois e 24 horas após a Maratona Internacional de São Paulo.

\begin{tabular}{c|c|c|c|c|c|c|c|c}
\hline & & \multicolumn{2}{|c|}{ Pré } & \multicolumn{2}{c|}{ Pós } & \multicolumn{2}{|c|}{ 24h } & \multirow{2}{*}{ Valor de $\mathbf{p}^{\text {AN }}$} \\
\hline & & $\mathrm{N}$ & $\%$ & $\mathrm{n}$ & $\%$ & $\mathrm{n}$ & $\%$ & \\
\hline $\begin{array}{c}\text { Troponina } \\
\text { (ng/mL) }\end{array}$ & Normal & 36 & 94,7 & 25 & 65,8 & 35 & 92,1 & \multirow{2}{*}{0,0010} \\
\hline & Alterada & 2 & 5,3 & 13 & 44,2 & 3 & 7,9 & \\
\hline \multirow{2}{*}{$\begin{array}{c}\text { BNP } \\
\text { (pg/mL) }\end{array}$} & Normal & 20 & 52,5 & - & - & & & \multirow{2}{*}{$<0,0001$} \\
\hline & Alterada & 18 & 47,4 & 38 & 100 & & & \\
\hline \multirow{2}{*}{ BNP ref } & Normal & 37 & 97,4 & 31 & 81,6 & & & \multirow{2}{*}{0,0258} \\
\cline { 2 - 7 } & Alterada & 1 & 2,6 & 7 & 18,4 & & & \multirow{2}{*}{} \\
\hline
\end{tabular}

Abreviações: Pré, pré maratona; pós, pós maratona; 24 h, 24 horas após a maratona; AN: ANOVA não paramétrica; Para troponina: $\mathrm{p}$ (pré $\mathrm{x}$ pós) $=0,0005 ; \mathrm{p}$ (pós $\times 24 h)<0,0001 ; \mathrm{p}$ (pré $\times 24 h$ ) $=0,5672$.

\section{DISCUSSÃO}

Os resultados do presente estudo indicam que a realização de uma maratona promoveu aumento significativo dos marcadores BNP e troponina T cardíaca em uma parcela considerável dos sujeitos da amostra.

O aumento do BNP foi observado em 18,4\% dos participantes enquanto, o aumento da troponina, foi verificado em 34\% dos atletas. Entretanto, o aumento de BNP não está relacionado com a indução da liberação de troponina T cardíaca pelo exercício, fato que está em concordância com estudos prévios, e sugere que a liberação destes dois biomarcadores de injúria miocárdica ocorram por mecanismos diferentes, apesar da indução ocorrer devido a realização de exercícios de longa duração ${ }^{10,16}$.

A liberação de troponinas cardíacas após exercício prolongado é documentada, sendo a alta prevalência de liberação de cTnT após o exercício de longa duração neste estudo consistente com estudos prévios com exercício de endurance.

A associação do exercício de endurance com uma transitória e pequena quantidade de troponina liberada, a qual na maioria dos casos tem a normalização dos níveis 24 horas depois da competição, corrobora com os achados da literatura ${ }^{17-19}$. Uma metanálise de 26 estudos demonstrou que ocorre elevação da troponina em aproximadamente $50 \%$ dos participantes de provas de endurance $e^{20}$. Desta forma, a maioria dos estudos mostra aumentos significativos de troponina cardíaca em grupos heterogêneos de atletas após completar uma maratona ou ultramaratona, positivamente relacionados a distância da competição realizada ${ }^{8,21}$.

Outros estudos analisaram as características dos sujeitos, do exercí- 
cio e o status de hidratação na liberação de troponinas cardíacas após exercícios de longa duração 22,23 .

Embora, a influência da experiência de treino, em jovens atletas, tenha sido apontado como fator importante e independente para a liberação de troponinas, principalmente ao considerar que em indivíduos mais jovens, para uma dada carga de trabalho, o miocárdio mais imaturo é mais vulnerável a injúria quando comparado com adultos, enquanto volume sistólico e débito cardíaco são menores. Entretanto, esses achados não foram confirmados no presente estudo ${ }^{22,23}$.

Durante a realização de exercícios com menor duração (5km), indivíduos não treinados têm aumentos significantes na concentração de troponina cardíaca T, em contraste aos indivíduos treinados. Entretanto, quando comparados com indivíduos que realizam exercícios extenuantes, essa liberação tem menor magnitude, concordante com o verificado na presente pesquisa, quando verificou-se que o tempo de prova tem relação com a liberação de troponinas após exercícios, demonstrando que houve uma maior demanda daquele miocárdio menos adaptado que foi exigido por um maior período de tempo 21,24.

A liberação de troponina decorrente de dano patológico pode ser dividida em três categorias: lesão isquêmica cardíaca primária, descrevendo a liberação de troponina devido à injúria causada pela ruptura de placa arterial coronariana e oclusão coronária; lesão isquêmica cardíaca secundária, que descreve isquemia miocárdica com injúria dos cardiomiócitos e ausência de ruptura de placa aterosclerótica derivadas do aumento de demanda miocárdica de oxigênio que supera o suprimento de oxigênio miocárdico e, lesão cardíaca não isquêmica, diagnosticada através da liberação de troponina causada por dano direto ao miocárdio.

Tendo como base esses três mecanismos, a liberação de troponina decorrente do exercício de endurance em indivíduos saudáveis, como mostrado em nosso estudo, ainda não pode ser explicado.

Nesse estudo verificou-se, 24 horas após a realização da maratona, uma redução significativa dos níveis de troponina em relação aos níveis imediatamente após a prova, demonstrando que a cinética e o tempo de elevação da troponina após maratona difere da cinética e o tempo de liberação desse marcador nas síndromes coronarianas agudas (SCA ${ }^{25}$.

Nas SCA a troponina é liberada do miocárdio para a circulação durante as primeiras poucas horas depois do início da isquemia de forma bifásica. Uma pequena liberação inicial é seguida por uma liberação sustentada maior com pico na concentração sérica de 6 a 12 horas $^{26}$. Portanto, cerca de 24 horas após a injúria miocárdica, na SCA encontram-se os maiores níveis de troponina circulantes, achado que não observamos após o exercício de endurance $e^{22,27}$. Desta forma, devido ao padrão de liberação de troponinas após exercício de longa duração, sugere-se fadiga cardíaca reversível no lugar de uma injúria cardíaca irreversível.

Porém, até o presente momento a etiologia da liberação de troponina cardíaca em sujeitos saudáveis após exercício de endurance ainda é desconhecida. A possível explicação para que os níveis de troponinas não estivessem elevados em todos os maratonistas pode incluir os diferentes níveis de treinamento dos participantes, a duração do exercício e a intensidade relativa do exercício ${ }^{28}$.

Os potenciais mecanismos que favorecem a liberação de troponina cardíaca, durante o exercício, podem estar relacionados à redução do clearance renal de forma aguda. Esse fato pode levar ao prolongamento do catabolismo de proteínas plasmáticas liberadas transitoriamente pelo cardiomiócitos que teria influência no metabolismo das troponinas cardíacas.

Outros mecanismos e etiologias aceitos são: a necrose dos cardiomiócitos, principalmente, por dano irreversível; mudança reversível e transitória da permeabilidade da membrana dos cardiomiócitos, em função do estresse mecânico, com rápida liberação de troponina do pool não ligado no citoplasma dos cardiomiócitos refletindo um processo fisiológico ao invés de patológico, aumento da produção de radicais livres ou balanço ácido-base alterado ${ }^{29}$.

As troponinas cardíacas são alvos das caspases e calpaínas, as quais são ativadas e liberadas durante a morte celular. Portanto, é esperado que as troponinas cardíacas sejam liberadas parcialmente fragmentadas quando originadas de células com dano irreversível, enquanto que quando a liberação tratar-se do citosol depois de danos reversíveis, elas devem estar predominantemente intactas ${ }^{30}$. Em análises in vitro foi demonstrado que há liberação de troponinas cardíacas intactas de cardiomiócitos estimulados em hipóxia sem morte celular ${ }^{31,32}$.

Diante do exposto, uma hipótese a ser considerada é a ocorrência de uma isquemia miocárdica transitória durante o exercício, devido a prioridade do fluxo sanguíneo para a musculatura esquelética. Nesse sentido, a ideia de que a isquemia miocárdica pode causar liberação de troponina já foi sustentada por estudos prévios e deve ser considerada na prática clínica e no exercício ${ }^{30}$.

É possível observar que a significância clínica do aumento dos marcadores cardíacos após exercícios de longa duração em praticantes saudáveis ainda não está totalmente estabelecida ${ }^{10}$, sendo que o mecanismo e magnitude da liberação de troponina parece ser multifatorial, tendo influência do tempo de exercício e até experiência do praticante. Alguns estudos acharam correlação da intensidade do exercício com a liberação de troponina, situação não observada em nosso estudo 7,22.

Em contraste à troponina cardíaca que indica danos nas células miocárdicas, o BNP é um biomarcador indicativo de sobrecarga miocárdica funcional e reflete o estresse da parede miocárdica. Quanto ao aumento dos níveis de BNP após exercício, nosso estudo corrobora dados da literatura que mostram aumento transitório deste marcador após exercício de endurance. Apesar de todos os atletas terem o BNP alterado após maratona, somente sete tiveram os valores acima do valor de corte de referência. Alguns estudos recentes mostraram aumentos não significativos em BNP pós exercício, desta forma, a resposta do BNP ao exercício em indivíduos saudáveis permanece controversa'.

Ohba et al.' foram os primeiros a examinar a resposta do BNP após exercício prolongado, e propuseram que este fenômeno poderia estar baseado em necrose subclínica de células miocárdicas.

Atualmente, os estudos mais recentes não dão suporte a esta hipótese $^{12}$, sugerindo que fatores como a massa do coração, sobrecarga de volume, estiramento das fibras musculares, aspectos hemodinâmicos relacionados com a pré e pós carga, assim como neuro-hormônios, possam contribuir para os aumentos de BNP induzidos pelo exercício. Isso porque acredita-se que o aumento do BNP possa servir como um mecanismo cardioprotetor e compensatório contra deterioração ou efeito da regulação do crescimento miocárdico.

Diante do exposto, argumenta-se que os níveis endógenos de BNP possam realizar um papel fisiológico em facilitar a performance cardiovascular durante o exercício, além de ser particularmente importante na insuficiência cardíaca diastólica ${ }^{33}$

Outra linha identificada e mais exaustivamente estudada modernamente, está relacionada ao fato da liberação do BNP ter um papel local direto anti-hipertrófico e poder estar relacionada a interação com vias específicas de sinalização pró-hipertróficas, sendo vista atualmente com papel ativo no processo de remodelamento cardíaco ${ }^{34}$.

Assim, é possível sugerir que o aumento nos níveis de BNP seja uma resposta fisiológica aos sinais de hipertrofia do coração e, portanto, estaria fundamentalmente relacionado com a duração do exercício e com o nível de treinamento do atleta, hipótese que corrobora os achados do nosso estudo, quando verificou-se a correlação entre o tempo de conclusão da maratona e os níveis de BNP imediatamente 
pós maratona assim como da diferença entre pré e pós prova porém, contrastando com outros estudos ${ }^{8}$.

Desta forma, a liberação de BNP aconteceria em resposta ao trabalho cardíaco e ao alongamento do tecido miocárdico independentemente da intensidade do exercício, podendo estar relacionado a um grau de disfunção cardíaca causado pelo aumento no estresse da parede miocárdica $2,6,11,16,35$. De acordo com Bird et al., ${ }^{36}$ o que determina a magnitude do estresse fisiológico produzido pelo exercício e as respostas agudas resultantes é a combinação da intensidade do exercício e as demandas do exercício relativas a condição física de cada indivíduo, sendo que o mesmo tipo de exercício pode produzir diferentes magnitudes de respostas e mudanças dos biomarcadores em indivíduos com diferentes níveis de treinamento.

Não encontramos correlação entre os valores de BNP e troponina após a maratona, apesar da correlação moderada com o tempo de prova para ambos os marcadores. Nesse sentido, nossos achados diferem de Klapcinska et a ${ }^{\beta 7}$ que encontraram correlação moderada dos valores desses biomarcadores entre si e com a velocidade de corrida, após uma ultramaratona de 24 horas $^{7,37}$.

\section{REFERÊNCIAS}

1. Ohba H, Takada H, Musha H, Nagashima J, Mori N, Awaya T, et al. Effects of prolonged strenuous exercise on plasma levels of atrial natriuretic peptide and brain natriuretic peptide in healthy men. Am Heart J. 2001;141(5):751-8.

2. Aagaard P, Sahlen A, Braunschweig F. Performance trends and cardiac biomarkers in a 30-km cross-country race, 1993-2007. Med Sci Sports Exerc. 2012;44(5):894-9.

3. Salgado JVV, Chacon-Mikahil M. Corrida de rua: análise do crescimento do número de provas e de praticantes. Conexões. 2006;4(1):90-9.

4. Roth HJ, Leithauser RM, Doppelmayr H, Doppelmayr M, Finkernagel H, von Duvillard SP, et al. Cardiospecificity of the $3 \mathrm{rd}$ generation cardiac troponin T assay during and after a $216 \mathrm{~km}$ ultra-endurance marathon run in Death Valley. Clin Res Cardiol. 2007;96(6):359-64.

5. Bachi AL, Rios FJ, Vaisberg PH, Martins M, de Sa MC, Victorino AB, et al. Neuro-immuno-endocrine modulation in marathon runners. Neuroimmunomodulation. 2015;22(3):196-202.

6. Kim YJ, Shin YO, Lee JB, Lee YH, Shin KA, Kim AC, et al. The effects of running a 308 km ultra-marathon on cardiac markers. Eur J Sport Sci. 2014;14(Suppl 1):S92-7.

7. Legaz-Arrese A, George K, Carranza-Garcia LE, Munguia-Izquierdo D, Moros-Garcia T, Serrano-Ostariz E. The impact of exercise intensity on the release of cardiac biomarkers in marathon runners. Eur J Appl Physiol. 2011;111(12):2961-7.

8. Eijsvogels TM, Hoogerwerf MD, Maessen MF, Seeger JP, George KP, Hopman MT, et al. Predictors of cardiac troponin release after a marathon. J Sci Med Sport. 2015;18(1):88-92.

9. Konig D, Schumacher YO, Heinrich L, Schmid A, Berg A, Dickhuth HH. Myocardial stress after competitive exercise in professional road cyclists. Med Sci Sports Exerc. 2003;35(10):1679-83.

10. Scharhag J, Urhausen A, Schneider G, Herrmann M, Schumacher K, Haschke M, et al. Reproducibility and clinical significance of exercise-induced increases in cardiac troponins and $\mathrm{N}$-terminal pro brain natriuretic peptide in endurance athletes. Eur J Cardiovasc Prev Rehabil. 2006;13(3):388-97.

11. Huang WS, Lee MS, Perng HW, Yang SP, Kuo SW, Chang HD. Circulating brain natriuretic peptide values in healthy men before and after exercise. Metabolism. 2002;51(11):1423-6.

12. Scharhag J, Herrmann M, Urhausen A, Haschke M, Herrmann W, Kindermann W. Independent elevations of $\mathrm{N}$-terminal pro-brain natriuretic peptide and cardiac troponins in endurance athletes after prolonged strenuous exercise. Am Heart J. 2005;150(6):1128-34.

13. Karlstedt E, Chelvanathan A, Da Silva M, Cleverley K, Kumar K, Bhullar N, et al. The impact of repeated marathon running on cardiovascular function in the aging population. J Cardiovasc Magn Reson. 2012;14:58.

14. Siegel S. Nonparametric statistics for the behavioral sciences. 1956.

15. Brunner E, Langer F. Nonparametric analysis of ordered categorical data in designs with longitudinal observations and small sample sizes. Biometrical Journal. 2000;42(6):663-75.

16. Wilson M, O'Hanlon R, Prasad S, Oxborough D, Godfrey R, Alpendurada F, et al. Biological markers of cardiac damage are not related to measures of cardiac systolic and diastolic function using cardiovascular magnetic resonance and echocardiography after an acute bout of prolonged endurance exercise. Br J Sports Med. 2011;45(10):780-4.

17. Lowbeer C, Seeberger A, Gustafsson SA, Bouvier F, Hulting J. Serum cardiac troponin T, troponin I, plasma BNP and left ventricular mass index in professional football players. J Sci Med Sport. 2007;10(5):291-6.

18. Neumayr G, Pfister R, Mitterbauer G, Eibl G, Hoertnagl H. Effect of competitive marathon cycling on plasma $\mathrm{N}$-terminal pro-brain natriuretic peptide and cardiac troponin $\mathrm{T}$ in healthy recreational cyclists. Am J Cardiol. 2005;96(5):732-5.

\section{CONCLUSÃO}

Os achados deste estudo sugerem que exercício de endurance está relacionado com a liberação dos marcadores cTnT e BNP, contudo, não refletindo uma situação clínica de injúria do coração, mas sim, um alto estresse temporário na parede miocárdica. Entretanto, ainda não é possível inferir o real significado clínico desta adaptação fisiológica. Mais estudos são necessários para melhor esclarecer os mecanismos e significados clínicos do aumento destes biomarcadores em decorrência dos exercícios de endurance.

\section{AGRADECIMENTOS}

Agradecemos ao Laboratório de Análises Clínicas do Instituto Dante Pazzanese de Cardiologia, assim como a empresa TEB - Tecnologia Eletrônica Brasileira e Yescom, produtora da Maratona Internacional de São Paulo, pelo apoio e auxilio neste projeto.

Todos os autores declararam não haver qualquer potencial conflito de interesses referente a este artigo.
19. O'Hanlon R, Wilson M, Wage R, Smith G, Alpendurada FD, Wong J, et al. Troponin release following endurance exercise: is inflammation the cause? a cardiovascular magnetic resonance study. J Cardiovasc Magn Reson. 2010;12:38

20. Passaglia DG, Emed LG, Barberato SH, Guerios ST, Moser Al, Silva MM, et al. Acute effects of prolonged physical exercise: evaluation after a twenty-four-hour ultramarathon. Arq Bras Cardiol. 2013;100(1):21-8.

21. Mingels AM, Jacobs LH, Kleijnen VW, Laufer EM, Winkens B, Hofstra L, et al. Cardiac troponin T elevations, using highly sensitive assay, in recreational running depend on running distance. Clin Res Cardiol. 2010;99(6):385-91

22. Mehta R, Gaze D, Mohan S, Williams KL, Sprung V, George K, et al. Post-exercise cardiac troponin release is related to exercise training history. Int J Sports Med. 2012;33(5):333-7.

23. Nie J, Tong TK, Shi Q, Lin H, Zhao J, Tian Y. Serum cardiac troponin response in adolescents playing basketball. Int J Sports Med. 2008;29(6):449-52.

24. Shave R, George KP, Atkinson G, Hart E, Middleton N, Whyte G, et al. Exercise-induced cardiac troponin T release: a meta-analysis. Med Sci Sports Exerc. 2007;39(12):2099-106.

25. Trivax JE, Franklin BA, Goldstein JA, Chinnaiyan KM, Gallagher MJ, deJong AT, et al. Acute cardiac effects of marathon running. J Appl Physiol (1985). 2010;108(5):1148-53.

26. Shave R, Ross P, Low D, George K, Gaze D. Cardiac troponin I is released following high-intensity short-duration exercise in healthy humans. Int J Cardiol. 2010;145(2):337-9.

27. Traiperm N, Gatterer H, Wille M, Burtscher M. Cardiac troponins in young marathon runners. Am . Cardiol. 2012;110(4):594-8.

28. O'Keefe JH, Patil HR, Lavie CJ, Magalski A, Vogel RA, McCullough PA. Potential adverse cardiovascular effects from excessive endurance exercise. Mayo Clin Proc. 2012;87(6):587-95.

29. Hubble KM, Fatovich DM, Grasko JM, Vasikaran SD. Cardiac troponin increases among marathon runners in the Perth Marathon: the Troponin in Marathons (TRIM) study. Med J Aust. 2009;190(2):91-3.

30. Streng AS, Jacobs LH, Schwenk RW, Cardinaels EP, Meex SJ, Glatz JF, et al. Cardiac troponin in ischemic cardiomyocytes: intracellular decrease before onset of cell death. Exp Mol Pathol. 2014;96(3):339-45.

31. Hessel MH, Atsma DE, van der Valk EJ, BaxWH, Schalij MJ, van der Laarse A. Release of cardiac troponin I from viable cardiomyocytes is mediated by integrin stimulation. Pflugers Arch. 2008;455(6):979-86.

32. Hessel MH, Michielsen EC, Atsma DE, Schalij MJ, van der Valk EJ, Bax WH, et al. Release kinetics of intact and degraded troponin I and T after irreversible cell damage. Exp Mol Pathol. 2008;85(2):90-5

33. Mottram PM, Haluska BA, MarwickTH. Response of B-type natriuretic peptide to exercise in hypertensive patients with suspected diastolic heart failure: correlation with cardiac function, hemodynamics, and workload. Am Heart J. 2004;148(2):365-70.

34. Calvieri C, Rubattu S, Volpe M. Molecular mechanisms underlying cardiac antihypertrophic and antifibrotic effects of natriuretic peptides. J Mol Med (Berl). 2012;90(1):5-13.

35. Shave R, Oxborough D. Exercise-induced cardiac injury: evidence from novel imaging techniques and highly sensitive cardiac troponin assays. Prog Cardiovasc Dis. 2012;54(5):407-15.

36. Bird SR, Linden M, Hawley JA. Acute changes to biomarkers as a consequence of prolonged strenuous running. Ann Clin Biochem. 2014;51(Pt 2):137-50.

37. Klapcinska B, Waskiewicz Z, Chrapusta SJ, Sadowska-Krepa E, CZuba M, Langfort J. Metabolic responses to a 48-h ultra-marathon run in middle-aged male amateur runners. Eur J Appl Physiol. 2013;113(11):2781-93 